\title{
Thermodynamic limit of the Nekrasov-type formula for E-string theory
}

\section{Takenori Ishii and Kazuhiro Sakai}

Department of Physical Sciences, College of Science and Engineering, Ritsumeikan University, 1-1-1 Noji-higashi, Kusatsu, Shiga 525-8577, Japan

E-mail: gr0054vr@ed.ritsumei.ac.jp, ksakai@fc.ritsumei.ac.jp

ABSTRACT: We give a proof of the Nekrasov-type formula proposed by one of the authors for the Seiberg-Witten prepotential for the E-string theory on $\mathbb{R}^{4} \times T^{2}$. We take the thermodynamic limit of the Nekrasov-type formula following the example of NekrasovOkounkov and reproduce the Seiberg-Witten description of the prepotential. The SeibergWitten curve obtained directly from the Nekrasov-type formula is of genus greater than one. We find that this curve is transformed into the known elliptic curve by a simple map. We consider the cases in which the low energy theory has $E_{8}, E_{7} \oplus A_{1}$ or $E_{6} \oplus A_{2}$ as a global symmetry.

KEYwords: Supersymmetric gauge theory, Matrix Models, Field Theories in Higher Dimensions

ARXIV EPRINT: 1312.1050 


\section{Contents}

1 Introduction 1

2 Seiberg-Witten prepotential and Nekrasov-type formula 2

3 Proof 5

3.1 Saddle point equation and resolvent 5

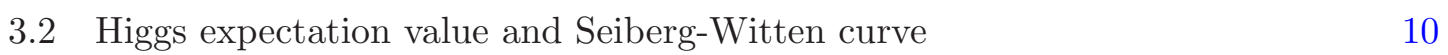

$\begin{array}{lll}3.3 & \text { Dual Higgs expectation value and prepotential } & 11\end{array}$

4 Cases with other global symmetries $\quad 13$

$\begin{array}{lll}4.1 & E_{7} \oplus A_{1} \text { theory } & 13\end{array}$

$\begin{array}{lll}4.2 E_{6} \oplus A_{2} \text { theory } & 13\end{array}$

5 Discussion $\quad 14$

$\begin{array}{ll}\text { A Conventions of special functions } & 15\end{array}$

\section{Introduction}

The E-string theory is probably the simplest interacting non-gravitational theory with $(1,0)$ supersymmetry in six dimensions $[1,2]$. The theory is obtained as the low energy theory of an M5-brane near one of the two fixed 9-planes in the heterotic M-theory. In the Coulomb branch it is a theory of just one tensor multiplet. Toroidal compactification of the theory exhibits rich structures [3-5]. When compactified down to four dimensions, the low energy effective theory is given by an $\mathcal{N}=2 \mathrm{U}(1)$ gauge theory which is fully characterized by the Seiberg-Witten solution [5-7]. See [8-12] for recent developments.

In $[13,14]$ a Nekrasov-type formula for the Seiberg-Witten prepotential for the E-string theory was proposed. It was also pointed out that the formula can be regarded as a special case of the elliptic generalization of a certain Nekrasov partition function $[15,16]$. The Nekrasov formula for ordinary gauge theories has been proved [17, 18]. More specifically, it has been shown that the prepotential obtained from the Nekrasov partition function is identical to that prescribed in terms of the Seiberg-Witten curve. It is natural to expect that the Nekrasov-type formula for the E-string theory can be proved in a similar manner. In this paper, we follow the example of Nekrasov and Okounkov [17] and give a proof of the Nekrasov-type formula for the E-string theory.

It is important to note that interpretation of parameters in the Nekrasov-type formula for the E-string theory is quite different from the conventional one for ordinary gauge theories. For instance, the parameter which represents the IR gauge coupling in the case 
of conformal gauge theories is identified with the expectation value of the Higgs field in the low energy theory of the E-string theory. Because of this difference, a straightforward generalization of the proof by Nekrasov and Okounkov does not work for the E-string theory. We overcome this problem by introducing the antiderivative of the resolvent and deriving Higgs expectation values from it.

Another nontrivial point is that the Seiberg-Witten curve obtained directly from the Nekrasov-type formula is of genus greater than one. This is not a desired result because the known Seiberg-Witten curve for the E-string theory is of genus one. We resolve this mismatch by finding a simple map which transforms the former higher genus curve into the latter elliptic curve.

We mainly consider the simplest case, namely the case where the E-string theory is compactified on $T^{2}$ without Wilson line parameters. In this case the low energy theory preserves the original $E_{8}$ global symmetry. The proof can be generalized to the cases with nontrivial Wilson line parameters. As an illustration, we discuss two examples which have $E_{7} \oplus A_{1}$ or $E_{6} \oplus A_{2}$ as a global symmetry.

The organization of this paper is as follows. In section 2, we review the Seiberg-Witten description and the Nekrasov-type formula for the E-string theory on $\mathbb{R}^{4} \times T^{2}$. In section 3 , we present our proof. In section 4 , we consider the cases where $E_{7} \oplus A_{1}$ or $E_{6} \oplus A_{2}$ is a global symmetry. Section 5 is devoted to the discussion. In appendix A, we present the definitions of special functions and some useful identities.

\section{Seiberg-Witten prepotential and Nekrasov-type formula}

In this section we review the Seiberg-Witten description of the prepotential for the E-string theory on $\mathbb{R}^{4} \times T^{2}$ and its Nekrasov-type expression which we will prove in the next section.

The low-energy theory of the E-string theory on $\mathbb{R}^{4} \times T^{2}$ is an $\mathcal{N}=2 \mathrm{U}(1)$ gauge theory in four dimensions and admits a Seiberg-Witten description [19, 20]. The low energy effective action is fully characterized by a holomorphic function called the prepotential. The prepotential can be expressed in terms of the Seiberg-Witten curve. For the sake of simplicity here we restrict ourselves to the simplest case with trivial Wilson line parameters. This is the case where the $E_{8}$ global symmetry is kept intact under the torus compactification. We will simply call it the $E_{8}$ theory hereafter. Seiberg-Witten prepotential for this $E_{8}$ theory was investigated in detail in [21]. See [8] for detailed characterization of the prepotential including the cases with general Wilson line parameters.

The Seiberg-Witten curve for the $E_{8}$ theory is given by

$$
y^{2}=4 x^{3}-\frac{1}{12} E_{4}(\tau) u^{4} x-\frac{1}{216} E_{6}(\tau) u^{6}+4 u^{5} .
$$

Here $\tau$ is the complex modulus of the $T^{2}$ on which the E-string theory is compactified and $u$ is the coordinate of the Coulomb branch moduli space. $E_{2 n}(\tau)$ are the Eisenstein series (see appendix A). In the Seiberg-Witten description, the expectation values of the Higgs fields in the vector multiplet and the dual vector multiplet are respectively given by

$$
\varphi(u, \tau)=\frac{i}{4 \pi^{2}} \int d u \oint_{\tilde{\alpha}} \frac{d x}{y}, \quad \varphi_{\mathrm{D}}(u, \tau)=\frac{i}{4 \pi^{2}} \int d u \oint_{\tilde{\beta}} \frac{d x}{y} .
$$


Here one-cycles $\tilde{\alpha}, \tilde{\beta}$ of the elliptic curve $(2.1)$ are chosen so that

$$
\oint_{\tilde{\alpha}} \frac{d x}{y}=\frac{2 \pi}{u}+\mathcal{O}\left(\frac{1}{u^{2}}\right), \quad \oint_{\tilde{\beta}} \frac{d x}{y}=\frac{2 \pi \tau}{u}+\mathcal{O}\left(\frac{1}{u^{2}}\right)
$$

for large $u$. The integration constants of the integrals in $u$ are fixed in the way described below. Inverting the function $\varphi(u, \tau)$ one can express $\varphi_{\mathrm{D}}$ as a function in $\varphi$ and $\tau$. The prepotential for the E-string theory is then prescribed as

$$
\frac{\partial F_{0}}{\partial \varphi}=8 \pi^{3} i\left(\varphi_{\mathrm{D}}-\tau \varphi\right)+\text { const. }
$$

Here const. could be a function in $\tau$, but is a constant with respect to $\varphi$. Throughout this paper we regard $\tau$ as a fixed parameter rather than a variable. Integrating the above expression in $\varphi$, one obtains the prepotential. The normalizations and the integration 'constants' of $\varphi, \varphi_{\mathrm{D}}$ and $F_{0}$ are fixed so that the prepotential admits the following expansion

$$
F_{0}(\varphi, \tau)=\sum_{n=1}^{\infty} \sum_{k=0}^{\infty} N_{n, k} \sum_{m=1}^{\infty} \frac{1}{m^{3}} e^{2 \pi i m(n \varphi+k \tau)} .
$$

Here $N_{n, k}$ are integers. The first few of them are [3]

$$
\begin{array}{llll}
N_{1,0}=1, & N_{1,1}=252, & N_{1,2}=5130, & \cdots \\
N_{2,0}=0, & N_{2,1}=0, & N_{2,2}=-9252, & \cdots
\end{array}
$$

This expression reflects the fact that the prepotential can also be viewed as the BPS partition function of the E-string theory on $\mathbb{R}^{5} \times S^{1}$ as well as the genus zero topological string amplitude for the local $\frac{1}{2} \mathrm{~K} 3 . \quad N_{n, k}$ represent multiplicities of BPS states in the effective theory in five dimensions as well as those of rational curves in the local $\frac{1}{2} \mathrm{~K} 3$.

Next, we recall the Nekrasov-type formula proposed in [13, 14]. Let us start with introducing some notations. Let $E$ denote a two-dimensional torus $\mathbb{C} /(2 \pi \mathbb{Z}+2 \pi \tau \mathbb{Z})$ and $\omega_{k}(k=0,1,2,3)$ be half periods of the torus:

$$
\omega_{0}=0, \quad \omega_{1}=\pi, \quad \omega_{2}=-\pi-\pi \tau, \quad \omega_{3}=\pi \tau .
$$

Throughout this paper the Weierstrass elliptic function $\wp(z)$ is defined over the torus $E$, i.e. $\wp(z)=\wp(z ; 2 \pi, 2 \pi \tau)$. We let $\alpha, \beta$ denote two fundamental one-cycles of the torus $E$. They are chosen in such a way that

$$
\oint_{\alpha} d z=2 \omega_{1}=2 \pi, \quad \oint_{\beta} d z=2 \omega_{3}=2 \pi \tau .
$$

Physically, $E$ may be understood as the dual torus of the $T^{2}$ on which the E-string theory is compactified. Here 'dual' means that Wilson line parameters with respect to the directions in the $T^{2}$ take values on $E$.

Let $\boldsymbol{R}=\left(R_{1}, \ldots, R_{N}\right)$ denote an $N$-tuple of partitions. Each partition $R_{k}$ is a nonincreasing sequence of nonnegative integers

$$
R_{k}=\left\{\mu_{k, 1} \geq \mu_{k, 2} \geq \cdots \geq \mu_{k, \ell\left(R_{k}\right)}>\mu_{k, \ell\left(R_{k}\right)+1}=\mu_{k, \ell\left(R_{k}\right)+2}=\cdots=0\right\} .
$$


Here the number of nonzero $\mu_{k, i}$ is denoted by $\ell\left(R_{k}\right) . R_{k}$ is represented by a Young diagram. We let $\left|R_{k}\right|$ denote the size of $R_{k}$, i.e. the number of boxes in the Young diagram of $R_{k}$ :

$$
\left|R_{k}\right|:=\sum_{i=1}^{\infty} \mu_{k, i}=\sum_{i=1}^{\ell\left(R_{k}\right)} \mu_{k, i}
$$

Similarly, the size of $\boldsymbol{R}$ is denoted by

$$
|\boldsymbol{R}|:=\sum_{k=1}^{N}\left|R_{k}\right| .
$$

We let $R_{k}^{\vee}=\left\{\mu_{k, 1}^{\vee} \geq \mu_{k, 2}^{\vee} \geq \cdots\right\}$ denote the conjugate partition of $R_{k}$. We also introduce the notation

$$
h_{k, l}(i, j):=\mu_{k, i}+\mu_{l, j}^{\vee}-i-j+1,
$$

which represents the relative hook-length of a box at $(i, j)$ between the Young diagrams of $R_{k}$ and $R_{l}$.

We are now able to write down the Nekrasov-type formula. As discussed in $[13,14]$, the formula can be expressed in several different ways. For our present purposes it is convenient to express the formula as a special case of the elliptic generalization of the Nekrasov partition function for the $\mathrm{U}(N)$ gauge theory with $2 N$ fundamental matters $[15,16]$

$$
Z:=\sum_{\boldsymbol{R}}\left(-e^{2 \pi i \varphi}\right)^{|\boldsymbol{R}|} \prod_{k=1}^{N} \prod_{(i, j) \in R_{k}} \frac{\prod_{n=1}^{2 N} \vartheta_{1}\left(\frac{1}{2 \pi}\left(a_{k}-m_{n}+(j-i) \hbar\right), \tau\right)}{\prod_{l=1}^{N} \vartheta_{1}\left(\frac{1}{2 \pi}\left(a_{k}-a_{l}+h_{k, l}(i, j) \hbar\right), \tau\right)^{2}}
$$

Here the sum is taken over all possible partitions $\boldsymbol{R}$ (including the empty partition). A set of indices $(i, j)$ run over the coordinates of all boxes in the Young diagram of $R_{k} \cdot \vartheta_{1}(z, \tau)$ is the Jacobi theta function (see appendix A). For consistency we require

$$
2 \sum_{k=1}^{N} a_{k}-\sum_{n=1}^{2 N} m_{n}=0
$$

where the equality should be regarded modulo periods of the torus $E$. To obtain the prepotential for the E-string theory with four general Wilson line parameters, we set

$$
N=4, \quad a_{k}=\omega_{k-1} \quad(k=1,2,3,4), \quad m_{n}=-m_{n+4} \quad(n=1,2,3,4) .
$$

The Seiberg-Witten prepotential for the E-string theory is then given by

$$
F_{0}=\left.\left(2 \hbar^{2} \ln Z\right)\right|_{\hbar=0} .
$$

The case of the $E_{8}$ theory is realized by simply setting all the Wilson line parameters $m_{n}$ to be zero. Actually, in this case one can simplify the expression and express $Z$ as a sum over three partitions [14]. More specifically, $Z$ for the $E_{8}$ theory is given by (2.13) with

$$
N=3, \quad a_{k}=\omega_{k} \quad(k=1,2,3), \quad m_{n}=0 \quad(n=1, \ldots, 6) .
$$

We will use this simplified form in the proof below. 
An important remark is that identification of parameters for the E-string theory is quite different from what is known for ordinary gauge theories. In the case of ordinary gauge theories, $a_{k}$ represent diagonal elements of the expectation value of the Higgs field and $\varphi$ represents the IR gauge coupling. On the other hand, for the E-string theory $a_{k}$ are set to fixed values as above and $\varphi$ represents the Higgs expectation value. $\tau$ is the complex modulus of the $T^{2}$ and it plays the role of the IR gauge coupling in the low energy theory in four dimensions. Because of this difference, a straightforward generalization of the proof by Nekrasov-Okounkov [17] does not work in the case of E-string theory. We will present a resolution to this problem in the next section.

\section{Proof}

In this section we prove that the prepotential given by the Nekrasov-type formula in the last section is equivalent to that expressed in terms of the Seiberg-Witten curve. Our proof consists of three parts. In subsection 3.1, we first take the thermodynamic limit of the sum over partitions (2.13) and express the prepotential as the solution of an extremum problem. In subsection 3.2, we derive the expression of the Higgs expectation value in terms of the Seiberg-Witten curve. In subsection 3.3, we show that the prepotential obtained in the thermodynamic limit is indeed equivalent to that expressed in the Seiberg-Witten description.

In the proof below we restrict ourselves to the case of the $E_{8}$ theory and eventually set parameters to the specific values given in (2.17). However, we prolong fixing these parameters until the very end, anticipating the generalization to the cases with nontrivial Wilson line parameters.

\subsection{Saddle point equation and resolvent}

Following the example of Nekrasov and Okounkov [17], we take the thermodynamic limit of the Nekrasov-type formula presented in the last section. What we need to do is to consider the thermodynamic limit $\hbar \rightarrow 0$ of the sum over partitions (2.13) and evaluate the prepotential (2.16). This problem has already been solved by Hollowood, Iqbal and Vafa [15]. However, in addition to their results we need the precise form of the antiderivative of the resolvent and its analytic properties, which are in fact essential to our proof. In this subsection we present a self-contained solution to the problem with emphasis on the new ingredients.

Let us start with introducing a function $\gamma(z ; \hbar)$ which satisfies the difference equation

$$
\gamma(z+\hbar ; \hbar)+\gamma(z-\hbar ; \hbar)-2 \gamma(z ; \hbar)=\ln \vartheta_{1}\left(\frac{z}{2 \pi}\right)
$$

and has the expansion

$$
\gamma(z ; \hbar)=\sum_{g=0}^{\infty} \hbar^{2 g-2} \gamma_{g}(z)
$$


The explicit form of $\gamma(z ; \hbar)$ is not important here. In the following we merely use the fact that

$$
\gamma_{0}^{\prime \prime}(z)=\ln \vartheta_{1}\left(\frac{z}{2 \pi}\right),
$$

which can be derived immediately by expanding the above difference equation in $\hbar$.

The summand of the main formula (2.13) is expressed as a finite product over boxes in Young diagrams. It is well known that a product of this kind can be rewritten as a formally infinite product. In the present case, (2.13) is rewritten as

$$
\begin{aligned}
& Z=\sum_{\boldsymbol{R}} e^{2 \pi i \tilde{\varphi}|\boldsymbol{R}|} Z_{\boldsymbol{R}}, \\
& Z_{\boldsymbol{R}}=\prod_{\substack{k, l=1 \\
(k, i) \neq(l, j)}}^{N} \prod_{\substack{i, j=1 \\
(j)}}^{\infty} \frac{\vartheta_{1}\left(\frac{1}{2 \pi}\left(a_{k}-a_{l}+\left(\mu_{k, i}-\mu_{l, j}+j-i\right) \hbar\right)\right)}{\vartheta_{1}\left(\frac{1}{2 \pi}\left(a_{k}-a_{l}+(j-i) \hbar\right)\right)} \\
& \quad \times \prod_{k=1}^{N} \prod_{n=1}^{2 N} \prod_{(i, j) \in R_{k}} \vartheta_{1}\left(\frac{1}{2 \pi}\left(a_{k}-m_{n}+(j-i) \hbar\right)\right),
\end{aligned}
$$

where

$$
\tilde{\varphi}:= \begin{cases}\varphi & \text { if } N \text { is odd }, \\ \varphi+\frac{1}{2} & \text { if } N \text { is even. }\end{cases}
$$

This form is more convenient for our present purposes. In the thermodynamic limit the typical size of the partition $\boldsymbol{R}$ contributing to the sum is very large and $Z$ may be expressed in terms of continuous profiles of partitions. Indeed, using the difference equation (3.1), one can verify that $Z_{\boldsymbol{R}}$ is expressed as

$$
\begin{aligned}
Z_{\boldsymbol{R}}=\exp \left[-\frac{1}{4} f d z d w f^{\prime \prime}(z) f^{\prime \prime}(w) \gamma(z\right. & -w ; \hbar)+\frac{1}{2} \sum_{n=1}^{2 N} f d z f^{\prime \prime}(z) \gamma\left(z-m_{n} ; \hbar\right) \\
& \left.+\sum_{k, l=1}^{N} \gamma\left(a_{k}-a_{l} ; \hbar\right)-\sum_{k=1}^{N} \sum_{n=1}^{2 N} \gamma\left(a_{k}-m_{n} ; \hbar\right)\right]
\end{aligned}
$$

Here $f(z)$ is the profile of the partition $\boldsymbol{R}$

$$
\begin{array}{r}
f(z)=\sum_{k=1}^{N}\left[\sum_{i=1}^{\ell\left(R_{k}\right)}\left(\left|z-a_{k}-\hbar\left(\mu_{k, i}-i+1\right)\right|-\left|z-a_{k}-\hbar\left(\mu_{k, i}-i\right)\right|\right)\right. \\
\left.+\left|z-a_{k}+\hbar \ell\left(R_{k}\right)\right|\right]
\end{array}
$$

and its second derivative is given by

$$
\begin{gathered}
f^{\prime \prime}(z)=2 \sum_{k=1}^{N}\left[\sum_{i=1}^{\ell\left(R_{k}\right)}\left(\delta\left(z-a_{k}-\hbar\left(\mu_{k, i}-i+1\right)\right)-\delta\left(z-a_{k}-\hbar\left(\mu_{k, i}-i\right)\right)\right)\right. \\
\left.+\delta\left(z-a_{k}+\hbar \ell\left(R_{k}\right)\right)\right]
\end{gathered}
$$




$$
\begin{aligned}
=2 \sum_{k=1}^{N}\left[\sum_{i=1}^{\infty}(\delta(z\right. & \left.-a_{k}-\hbar\left(\mu_{k, i}-i+1\right)\right)-\delta\left(z-a_{k}-\hbar\left(\mu_{k, i}-i\right)\right) \\
& \left.\left.-\delta\left(z-a_{k}+\hbar(i-1)\right)+\delta\left(z-a_{k}+\hbar i\right)\right)+\delta\left(z-a_{k}\right)\right] .
\end{aligned}
$$

For a partition of large size $f^{\prime \prime}(z)$ can be viewed as a density function. We consider the case where $f^{\prime \prime}(z)$ has $N$ local supports respectively around $z=a_{k}(k=1, \ldots, N)$ and all of them are entirely separated from each other. We let $\mathcal{C}_{k}$ denote the local support around $z=a_{k}$ and $\mathcal{C}$ denote their union, i.e. $\mathcal{C}=\bigcup_{k=1}^{N} \mathcal{C}_{k}$. It follows from the above expression that

$$
\begin{aligned}
a_{k} & =\frac{1}{2} \int_{\mathcal{C}_{k}} z f^{\prime \prime}(z) d z \\
|\boldsymbol{R}| & =\frac{1}{4} \int_{\mathcal{C}} d z z^{2} f^{\prime \prime}(z)-\sum_{k=1}^{N} \frac{a_{k}^{2}}{2} .
\end{aligned}
$$

In the thermodynamic limit, the sum over partition $Z$ can be approximated by an integral over the space of continuous functions $f^{\prime \prime}$

$$
Z \simeq \int \mathcal{D} f^{\prime \prime} d^{N} \lambda \exp \left[\frac{1}{2 \hbar^{2}} \mathcal{F}_{0}+\mathcal{O}\left(\hbar^{0}\right)\right]
$$

where $\mathcal{F}_{0}$ is a functional of the following form

$$
\begin{aligned}
\mathcal{F}_{0}\left[f^{\prime \prime}, \lambda_{k}\right]= & -\frac{1}{2} f_{\mathcal{C}} d z d w f^{\prime \prime}(z) f^{\prime \prime}(w) \gamma_{0}(z-w)+\sum_{n=1}^{2 N} f_{\mathcal{C}} d z f^{\prime \prime}(z) \gamma_{0}\left(z-m_{n}\right) \\
& +4 \pi i \tilde{\varphi}\left(\frac{1}{4} \int_{\mathcal{C}} d z z^{2} f^{\prime \prime}(z)-\sum_{k=1}^{N} \frac{a_{k}^{2}}{2}\right) \\
& +2 \sum_{k=1}^{N} \lambda_{k}\left(\frac{1}{2} \int_{\mathcal{C}_{k}} d z z f^{\prime \prime}(z)-a_{k}\right)
\end{aligned}
$$

We have introduced Lagrange multipliers $\lambda_{k}$ taking account of the constraints (3.10). The integral (3.12) can be evaluated by the saddle point approximation. The prepotential (2.16) is then given, up to a constant, by the extremum of the functional $\mathcal{F}_{0}$. Taking the variation of $\mathcal{F}_{0}$, one obtains the saddle point equation

$$
f_{\mathcal{C}} d w f^{\prime \prime}(w) \gamma_{0}(z-w)-\sum_{n=1}^{2 N} \gamma_{0}\left(z-m_{n}\right)-\pi i \tilde{\varphi} z^{2}-\lambda_{k} z=0, \quad z \in \mathcal{C}_{k} .
$$

Solving this equation with the constraints (3.10) and plugging the solution back into (3.13), one obtains the prepotential $F_{0}$.

To solve this equation, it is convenient to consider the following analytic function

$$
\begin{aligned}
\Omega(z) & :=\int_{\mathcal{C}} f^{\prime \prime}(w) \gamma_{0}^{\prime \prime}(z-w) d w-\sum_{n=1}^{2 N} \gamma_{0}^{\prime \prime}\left(z-m_{n}\right) \\
& =\int_{\mathcal{C}} f^{\prime \prime}(w) \ln \vartheta_{1}\left(\frac{z-w}{2 \pi}\right) d w-\sum_{n=1}^{2 N} \ln \vartheta_{1}\left(\frac{z-m_{n}}{2 \pi}\right)
\end{aligned}
$$


instead of $f^{\prime \prime}(z)$ itself. We call it the antiderivative of the resolvent, as its derivative

$$
\omega(z):=\Omega^{\prime}(z)
$$

plays the role of the resolvent. Indeed, the density function $f^{\prime \prime}$ is recovered as

$$
2 \pi i f^{\prime \prime}(z)=\omega(z-i \epsilon)-\omega(z+i \epsilon) \quad z \in \mathcal{C} .
$$

Here $\epsilon=\delta z$ is an infinitesimal deformation along the cuts, so that $\pm i \epsilon$ represent infinitesimal deviations transverse to the cuts. By definition the Riemann surface of $\Omega(z)$ has logarithmic branches. It follows that

$$
\oint_{\gamma_{k}} \omega(z) d z=4 \pi i, \quad \oint_{\gamma^{(n)}} \omega(z) d z=-2 \pi i,
$$

where $\gamma_{k}(k=1, \ldots, N)$ and $\gamma^{(n)}(n=1, \ldots, 2 N)$ denote cycles encircling counterclockwise the cut $\mathcal{C}_{k}$ and the pole at $z=m_{n}$ respectively.

In terms of $\Omega(z)$, the second derivative of the saddle point equation (3.14) is written as

$$
\frac{1}{2}(\Omega(z-i \epsilon)+\Omega(z+i \epsilon))-2 \pi i \tilde{\varphi}=0 \quad z \in \mathcal{C} .
$$

Let us solve this equation. While $\Omega(z)$ has logarithmic branch points as well as square root branch points, the following function

$$
G(z):=e^{\Omega(z)-2 \pi i \tilde{\varphi}}+e^{-\Omega(z)+2 \pi i \tilde{\varphi}}
$$

is a meromorphic function on $E$. Poles at $z=m_{n}(n=1, \ldots, 2 N)$ are the whole singularities of $G(z)$. Since (2.14) is imposed, $G(z)$ is strictly doubly periodic. In other words, $G(z)$ is an elliptic function of order $2 N$. In terms of $G(z), \omega(z)$ is expressed as

$$
\omega(z)=\frac{G^{\prime}(z)}{\sqrt{(G(z)+2)(G(z)-2)}} .
$$

Since $G(z) \pm 2$ are elliptic functions of order $2 N$ and have $2 N$ zeros, the above expression implies that $\omega(z)$ would generically have $4 N$ branch points. On the other hand, in our setup $\omega(z)$ actually has just $2 N$ branch points. The mismatch is resolved if the function

$$
H(z):=\frac{G(z)+2}{4}=\cosh ^{2}\left(\frac{1}{2}(\Omega(z)-2 \pi i \tilde{\varphi})\right)
$$

has $N$ zeros of multiplicity two instead of $2 N$ simple zeros. ${ }^{1}$ The singularities of $H(z)$ are the single poles at $z=m_{n}(n=1, \ldots, 2 N)$. Elliptic functions satisfying these properties are determined as

$$
H(z)=\kappa \frac{P(z)^{2}}{Q(z)}
$$

\footnotetext{
${ }^{1}$ In general, there are possibilities that $G+2$ and $G-2$ have respectively $N-l$ and $l$ zeros of multiplicity two $(0 \leq l \leq N)$. If parameters are chosen as $a_{k}, \hbar \in \mathbb{R}, \tilde{\varphi}, \tau \in i \mathbb{R}$ and $m_{n}=0$, all $\mathcal{C}_{k}$ have to lie on the real axis and $\exp \Omega(z)>0$ for any $z \in \mathbb{R}$ with $z \notin \mathcal{C}$. This means that only the solution with $l=0$ is allowed in this case. The parameter settings for the $E_{r} \oplus A_{8-r}(r=8,7,6)$ theories are connected with the above setup by a continuous deformation preserving the topology of the branch cut configuration of $\Omega(z)$. Thus, the solution with $l=0$ is singled out for these theories. (The solution with $l=N$ is also allowed, but this is essentially the same as the solution with $l=0$.)
} 
with

$$
P(z)=\prod_{k=1}^{N} \vartheta_{1}\left(\frac{z-\zeta_{k}}{2 \pi}\right), \quad Q(z)=\prod_{n=1}^{2 N} \vartheta_{1}\left(\frac{z-m_{n}}{2 \pi}\right),
$$

where $\kappa$ and $\zeta_{k}(k=1, \ldots, N)$ are some constants. The locations of zeros and poles have to satisfy

$$
2 \sum_{k=1}^{N} \zeta_{k}-\sum_{n=1}^{2 N} m_{n}=0
$$

Here the equality should be understood modulo periods of the torus $E$. From $(3.22) \Omega(z)$ is obtained as

$$
\Omega(z)=2 \ln (\sqrt{H(z)}+\sqrt{H(z)-1})+2 \pi i \tilde{\varphi} .
$$

By taking the derivative, the resolvent is obtained as

$$
\omega(z)=\frac{2 \partial_{z} \sqrt{H(z)}}{\sqrt{H(z)-1}} .
$$

Substituting (3.23) one can verify that this is essentially equivalent to the resolvent given in $[15]$.

Recall that $f^{\prime \prime}(z)$ has to satisfy the constraints (3.10). In terms of the resolvent, they are expressed as

$$
a_{k}=\frac{1}{4 \pi i} \oint_{\gamma_{k}} z \omega(z) d z .
$$

These equations hold if $\omega(z)$ satisfies

$$
\omega\left(a_{k}-z \pm i \epsilon\right)=\omega\left(a_{k}+z \pm i \epsilon\right) \quad \text { for } \quad a_{k}+z \in \mathcal{C}_{k} .
$$

This holds if the function $H^{1 / 2}(z):=\sqrt{H(z)}$ satisfies

$$
H^{1 / 2}\left(a_{k}-z\right)=-H^{1 / 2}\left(a_{k}+z\right) \quad \text { for } \quad a_{k}+z \in \mathcal{C}_{k} .
$$

By requiring this property, the values of $\zeta_{k}$ are fixed.

Let us now restrict ourselves to the $E_{8}$ theory by setting parameters as in (2.17). In this case, as we will see immediately, the condition (3.30) is satisfied with

$$
\zeta_{k}=\omega_{k} \quad(k=1,2,3) .
$$

By substituting these data, the functions $P(z), Q(z)$ are expressed as

$$
P(z)=-i q^{-1 / 4} \prod_{k=1}^{3} \vartheta_{k+1}\left(\frac{z}{2 \pi}\right), \quad Q(z)=\vartheta_{1}\left(\frac{z}{2 \pi}\right)^{6} .
$$

The function $H=\kappa P^{2} / Q$ is then obtained as

$$
H(z)=-\frac{1}{4} u \wp^{\prime}(z)^{2},
$$


where we have used the identity (A.13) and introduced

$$
u:=\frac{4 \kappa}{q^{1 / 2} \eta^{12}} .
$$

Using the property $\wp^{\prime}(-z)=-\wp^{\prime}(z)$ and the periodicity of $\wp^{\prime}(z)$ one can verify that the above $H(z)$ indeed possesses the property (3.30). The resolvent for the $E_{8}$ theory is then explicitly expressed as

$$
\omega(z)=\frac{2 \wp^{\prime \prime}(z)}{\sqrt{\wp^{\prime}(z)^{2}+4 u^{-1}}} .
$$

Using (3.17) and plugging the above solution back into (3.13), one obtains the integral expression for the prepotential. The Riemann surface of the above resolvent $\omega(z)$ has three cuts near $z=\omega_{k}(k=1,2,3)$. The three cuts shrink as $|u|$ increases. In particular, when $u$ is sent to infinity all cuts disappear and the Riemann surface of $\omega(z)$ becomes the torus $E$ with complex modulus $\tau$. This is reminiscent of the classical limit of the Seiberg-Witten curve (2.1). Indeed, the above $u$ is going to be identified with the coordinate of the Coulomb branch moduli space in the Seiberg-Witten description.

\subsection{Higgs expectation value and Seiberg-Witten curve}

In this subsection we express $\varphi$ in terms of the function $H(z)$ and reproduce the SeibergWitten description. To do this, we make use of the following fact

$$
\frac{1}{2 \pi^{2} i} \oint_{\alpha} \ln \vartheta_{1}\left(\frac{z-w}{2 \pi}\right) d z=C_{1}(\tau) \quad \bmod \mathbb{Z},
$$

where $C_{1}(\tau)$ is some function in $\tau$. The explicit form of $C_{1}(\tau)$ is not important. What is crucial here is that $C_{1}(\tau)$ is independent of $w$ and also invariant under continuous deformation of the integration contour. This fact can be shown as follows: since the theta function is quasi-periodic $\vartheta_{1}(z+1)=-\vartheta_{1}(z)$, function $\frac{1}{2 \pi i} \ln \vartheta_{1}\left(\frac{z-w}{2 \pi}\right)^{2}$ is single-valued modulo $\mathbb{Z}$ along a loop belonging to the cycle $\alpha$. Recall also that the theta function is regular for $|z|<\infty$, so that the integral is invariant under the continuous deformation of the loop.

Substituting (3.8) into (3.15) and using the above fact one sees that

$$
\frac{1}{4 \pi^{2} i} \oint_{\alpha} \Omega(z) d z=0 \quad \bmod \mathbb{Z}
$$

where $C_{1}$ 's cancel with each other. Substituting (3.26), one obtains

$$
\tilde{\varphi}=\frac{i}{2 \pi^{2}} \oint_{\alpha} \ln (\sqrt{H(z)}+\sqrt{H(z)-1}) d z \quad \bmod \mathbb{Z}
$$

This gives an explicit expression of $\varphi$ which is related to $\tilde{\varphi}$ by (3.5).

We now show that the above expression is equivalent to the known Seiberg-Witten description of $\varphi$. Differentiating the above expression in $u$ one obtains

$$
\frac{\partial \varphi}{\partial u}=\frac{i}{4 \pi^{2} u} \oint_{\alpha} \frac{d z}{\sqrt{1-H(z)^{-1}}} .
$$


In the case of the $E_{8}$ theory, it can be written as

$$
\frac{\partial \varphi}{\partial u}=\frac{i}{4 \pi^{2} u} \oint_{\alpha} \frac{\wp^{\prime}(z) d z}{\sqrt{\wp^{\prime}(z)^{2}+4 u^{-1}}}
$$

The Seiberg-Witten curve should be given as the Riemann surface of the integrand. It is made of two copies of the torus $E$ connected with each other by three cuts near $z=\omega_{k}(k=$ $1,2,3)$. Thus, the Riemann surface is of genus four. However, by using the identity (A.11) and changing the variables as

$$
\wp(z)=u^{-2} x
$$

one obtains

$$
\frac{\partial \varphi}{\partial u}=\frac{i}{4 \pi^{2}} \oint_{\tilde{\alpha}} \frac{d x}{y}
$$

where $y$ is given by

$$
y^{2}=4 x^{3}-\frac{1}{12} E_{4} u^{4} x-\frac{1}{216} E_{6} u^{6}+4 u^{5} .
$$

This is exactly the Seiberg-Witten curve (2.1) for the E-string theory! It is clear from the definitions (2.3), (2.8) that $\tilde{\alpha}$ is the image of $\alpha$ by the map (3.41). Thus, the above expression for $\varphi$ is in perfect agreement with the Seiberg-Witten description of the Higgs expectation value $(2.2)$.

\subsection{Dual Higgs expectation value and prepotential}

To complete our proof, we need to show that the prepotential obtained from the Nekrasovtype formula is also expressed in terms of period integrals as in (2.4) with (2.2). For this purpose we consider the contour integral of $\Omega(z)$ around the cycle $\beta$. To do this, we make use of the modular transformation law of the theta function

$$
\vartheta_{1}\left(\frac{z}{2 \pi}, \tau\right)=e^{3 \pi i / 4} \tau^{-1 / 2} \exp \left(-\frac{i z^{2}}{4 \pi \tau}\right) \vartheta_{1}\left(\frac{z}{2 \pi \tau},-\frac{1}{\tau}\right) .
$$

Using this and applying (3.36) with modulus $-1 / \tau$, one can show that

$$
\begin{aligned}
& \frac{1}{2 \pi^{2} i \tau} \int_{z_{0}}^{z_{0}+2 \pi \tau} \ln \vartheta_{1}\left(\frac{z-w}{2 \pi}, \tau\right) d z \\
&=-\frac{1}{8 \pi^{3} \tau^{2}} \int_{z_{0}}^{z_{0}+2 \pi \tau}(z-w)^{2} d z \\
&+\frac{1}{2 \pi^{2} i \tau} \int_{z_{0}}^{z_{0}+2 \pi \tau} \ln \vartheta_{1}\left(\frac{z-w}{2 \pi \tau},-\frac{1}{\tau}\right) d z+\frac{3}{4}-\frac{1}{2 \pi i} \ln \tau \\
&=-\frac{1}{8 \pi^{3} \tau^{2}} \int_{z_{0}}^{z_{0}+2 \pi \tau}(z-w)^{2} d z+C_{1}\left(-\frac{1}{\tau}\right)+\frac{3}{4}-\frac{1}{2 \pi i} \ln \tau \bmod \mathbb{Z} \\
&=-\frac{1}{4 \pi^{2} \tau} w^{2}+\left(\frac{1}{2 \pi}+\frac{z_{0}}{2 \pi^{2} \tau}\right) w+C_{2}\left(z_{0}, \tau\right) \bmod \mathbb{Z}
\end{aligned}
$$


where $C_{2}\left(z_{0}, \tau\right)$ is some function in $z_{0}$ and $\tau$. By using this together with (3.8) and (3.15), one obtains

$$
\begin{aligned}
\frac{1}{4 \pi^{2} i \tau} & \int_{z_{0}}^{z_{0}+2 \pi \tau} \Omega(z) d z \\
& =-\frac{1}{8 \pi^{2} \tau} f_{\mathcal{C}} w^{2} f^{\prime \prime}(w) d w+\left(\frac{1}{4 \pi}+\frac{z_{0}}{4 \pi^{2} \tau}\right) f_{\mathcal{C}} w f^{\prime \prime}(w) d w \quad \bmod \mathbb{Z} \\
& =\frac{i}{8 \pi^{3} \tau}\left(\frac{\partial F_{0}}{\partial \varphi}+2 \pi i \sum_{k=1}^{N} a_{k}^{2}\right)+\left(\frac{1}{2 \pi}+\frac{z_{0}}{2 \pi^{2} \tau}\right) \sum_{k=1}^{N} a_{k} \bmod \mathbb{Z} .
\end{aligned}
$$

$C_{2}$ 's cancel with each other in the first equality. To show the second equality we use (3.10) and (3.13) with

$$
\frac{\partial F_{0}}{\partial \varphi}=\left.\frac{\partial \mathcal{F}_{0}}{\partial \varphi}\right|_{\text {extremum }}=\left[\left(\frac{\partial \mathcal{F}_{0}}{\partial \varphi}\right)_{f^{\prime \prime}}+\left(\frac{\delta \mathcal{F}_{0}}{\delta f^{\prime \prime}}\right)_{\varphi} \frac{\partial f^{\prime \prime}}{\partial \varphi}\right]_{\text {extremum }}=\left[\left(\frac{\partial \mathcal{F}_{0}}{\partial \varphi}\right)_{f^{\prime \prime}}\right]_{\text {extremum }} .
$$

Here $\left(\partial \mathcal{F}_{0} / \partial \varphi\right)_{f^{\prime \prime}}$ denotes the partial derivative of $\mathcal{F}_{0}$ with respect to $\varphi$, holding $f^{\prime \prime}$ constant.

Let us now restrict ourselves to the $E_{8}$ theory. In this case the second term of the last line in (3.46) vanishes as we set $a_{k}=\omega_{k}(k=1,2,3){ }^{2}$ Thus, the integral is actually independent of $z_{0}$ and is regarded as the period integral over the cycle $\beta$. To sum up, one obtains

$$
\frac{1}{4 \pi^{2} i \tau} \oint_{\beta} \Omega(z) d z=\frac{i}{8 \pi^{3} \tau}\left(\frac{\partial F_{0}}{\partial \varphi}+2 \pi i \sum_{k=1}^{3} \omega_{k}^{2}\right) \quad \bmod \mathbb{Z}
$$

On the other hand, by using (3.26) the same period integral is expressed as

$$
\begin{aligned}
\frac{1}{4 \pi^{2} i \tau} \oint_{\beta} \Omega(z) d z & =\frac{1}{2 \pi^{2} i \tau} \oint_{\beta} \ln (\sqrt{H(z)}+\sqrt{H(z)-1}) d z+\tilde{\varphi} \\
& =-\frac{1}{\tau} \varphi_{\mathrm{D}}+\varphi+\text { const. }
\end{aligned}
$$

where we have identified the dual Higgs expectation value $\varphi_{\mathrm{D}}$ as

$$
\varphi_{\mathrm{D}}=\frac{i}{2 \pi^{2}} \oint_{\beta} \ln (\sqrt{H(z)}+\sqrt{H(z)-1}) d z+\text { const. }
$$

regarding the expression (3.38) for $\varphi$. Here const.'s are some functions in $\tau$ but are independent of $\varphi$. By comparing these two expressions, one obtains

$$
\frac{\partial F_{0}}{\partial \varphi}=8 \pi^{3} i\left(\varphi_{\mathrm{D}}-\tau \varphi\right)+\text { const. }
$$

This is in perfect agreement with the Seiberg-Witten description (2.4).

\footnotetext{
${ }^{2}$ The second term in (3.46) actually vanishes not only in the $E_{8}$ case but in most of the cases with generic four Wilson line parameters (2.15). On the other hand, it does not vanish in some special cases, such as the case of $E_{7} \oplus A_{1}$ symmetry, which we will study later. In these cases, the contour integral of $\Omega(z)$ along the cycle $\beta$ makes sense only up to a constant. Anyway, we will eventually show a relation up to a constant and thus such a constant ambiguity is irrelevant.
} 


\section{Cases with other global symmetries}

The proof presented in the last section can be generalized to the cases with nontrivial Wilson line parameters. As an illustration, we briefly discuss two examples which have $E_{7} \oplus A_{1}$ or $E_{6} \oplus A_{2}$ as a global symmetry.

\section{1 $\quad E_{7} \oplus A_{1}$ theory}

The case of $E_{7} \oplus A_{1}$ global symmetry is realized by setting the parameters as

$$
N=2, \quad a_{k}=\omega_{k+1} \quad(k=1,2), \quad m_{n}=0 \quad(n=1,2,3,4) .
$$

In this case, the condition (3.30) is satisfied if we choose $\zeta_{k}$ as

$$
\zeta_{k}=\omega_{k+1} \quad(k=1,2) .
$$

The function $H(z)$ is obtained as

$$
H(z)=\frac{u \vartheta_{3}^{2} \vartheta_{4}^{2}}{16} \frac{\wp^{\prime}(z)^{2}}{\wp(z)-e_{1}},
$$

where $u$ is defined as in (3.34) but with an opposite sign and

$$
e_{1}=\frac{\vartheta_{3}^{4}+\vartheta_{4}^{4}}{12}
$$

We have abbreviated $\vartheta_{k}(0, \tau)$ as $\vartheta_{k}$. Let us introduce a new variable $x$ by

$$
\wp(z)-e_{1}=u^{-2} x .
$$

One then obtains

$$
\frac{\partial \varphi}{\partial u}=\frac{i}{4 \pi^{2}} \oint_{\tilde{\alpha}} \frac{d x}{y}
$$

where $y$ is given by

$$
y^{2}=4 x^{3}+\left(\vartheta_{3}^{4}+\vartheta_{4}^{4}\right) u^{2} x^{2}+\left(\frac{\vartheta_{3}^{4} \vartheta_{4}^{4}}{4} u-\frac{16}{\vartheta_{3}^{2} \vartheta_{4}^{2}}\right) u^{3} x .
$$

This is exactly the Seiberg-Witten curve for the $E_{7} \oplus A_{1}$ case [14].

\section{$4.2 \quad E_{6} \oplus A_{2}$ theory}

The case of $E_{6} \oplus A_{2}$ global symmetry is realized by setting the parameters as

$$
N=3, \quad a_{k}=\omega_{k} \quad(k=1,2,3), \quad m_{n}=-m_{n+3}=\frac{2 \pi}{3} \quad(n=1,2,3) .
$$

In this case, the condition (3.30) is satisfied if we choose $\zeta_{k}$ as

$$
\zeta_{k}=\omega_{k} \quad(k=1,2,3) .
$$


Let us introduce the notation

$$
\alpha_{3}:=\vartheta_{3}(0,2 \tau) \vartheta_{3}(0,6 \tau)+\vartheta_{2}(0,2 \tau) \vartheta_{2}(0,6 \tau), \quad \beta_{3}:=\frac{\eta(\tau)^{9}}{\eta(3 \tau)^{3}} .
$$

By using the following identity

$$
\vartheta_{1}\left(\frac{z}{2 \pi}-\frac{1}{3}\right) \vartheta_{1}\left(\frac{z}{2 \pi}+\frac{1}{3}\right)=-3 \frac{\eta(3 \tau)^{2}}{\eta(\tau)^{6}}\left(\wp(z)-\frac{1}{4} \alpha_{3}^{2}\right) \vartheta_{1}\left(\frac{z}{2 \pi}\right)^{2}
$$

and (A.13), the function $H(z)$ is obtained as

$$
H(z)=\frac{u \beta_{3}^{2}}{108} \frac{\wp^{\prime}(z)^{2}}{\left(\wp(z)-\frac{1}{4} \alpha_{3}^{2}\right)^{3}} .
$$

Here $u$ is given by (3.34). Let us introduce a new variable $x$ by

$$
\wp(z)-\frac{1}{4} \alpha_{3}^{2}=\frac{x}{u\left(u-27 \beta_{3}^{-2}\right)} .
$$

One then obtains

$$
\frac{\partial \varphi}{\partial u}=\frac{i}{4 \pi^{2}} \oint_{\tilde{\alpha}} \frac{d x}{y}
$$

where $y$ is given by

$$
y^{2}=4 x^{3}+3 \alpha_{3}^{2} u^{2} x^{2}+\frac{2}{3} \alpha_{3}\left(\beta_{3} u-\frac{27}{\beta_{3}}\right) u^{3} x+\frac{1}{27}\left(\beta_{3} u-\frac{27}{\beta_{3}}\right)^{2} u^{4} .
$$

This is exactly the Seiberg-Witten curve for the $E_{6} \oplus A_{2}$ case [14].

\section{Discussion}

We have proved the Nekrasov-type formula for the Seiberg-Witten prepotential for the E-string theory on $\mathbb{R}^{4} \times T^{2}$. Following the example of Nekrasov-Okounkov, we have taken the thermodynamic limit of the Nekrasov-type formula and have determined the profile which dominates the saddle point approximation of the sum over partitions. Due to the difference in identification of parameters between the E-string theory and ordinary gauge theories, the proof by Nekrasov-Okounkov cannot be straightforwardly generalized. We have resolved this problem by considering the antiderivative of the resolvent rather than the resolvent itself in the thermodynamic limit.

The Seiberg-Witten curve obtained directly from the Nekrasov-type formula is of genus greater than one and is not an elliptic curve. We have found a simple transformation of variables by means of the Weierstrass $\wp$-function which maps the higher genus curve to the known elliptic Seiberg-Witten curve for the E-string theory. Such a simplification is possible because the parameters in the Nekrasov-type formula have been chosen specifically for the setup of the E-string theory.

As the E-string theory is one of the simplest non-Lagrangian field theories, the theory is ubiquitous in the study of such theories in higher dimensions. For instance, the five-dimensional limit of the E-string theory with $E_{6}$ global symmetry is identical to the $T_{3}$ theory in five dimensions [22], for which Nekrasov-type partition functions have been studied recently $[23,24]$. We hope that investigations into Nekrasov-type formulas for the E-string theory will shed light on the mysterious nature of non-Lagrangian field theories. 


\section{Acknowledgments}

The authors would like to thank T. Eguchi, S. Moriyama, K. Ohta and especially Y. Sugawara for helpful comments and discussions. The work of K.S. is supported in part by Grant-in-Aid for Scientific Research from the Ministry of Education, Culture, Sports, Science and Technology of Japan (MEXT).

\section{A Conventions of special functions}

The Jacobi theta functions are defined as

$$
\begin{aligned}
\vartheta_{1}(z, \tau) & :=i \sum_{n \in \mathbb{Z}}(-1)^{n} y^{n-1 / 2} q^{(n-1 / 2)^{2} / 2} \\
\vartheta_{2}(z, \tau) & :=\sum_{n \in \mathbb{Z}} y^{n-1 / 2} q^{(n-1 / 2)^{2} / 2} \\
\vartheta_{3}(z, \tau) & :=\sum_{n \in \mathbb{Z}} y^{n} q^{n^{2} / 2} \\
\vartheta_{4}(z, \tau) & :=\sum_{n \in \mathbb{Z}}(-1)^{n} y^{n} q^{n^{2} / 2}
\end{aligned}
$$

where $y=e^{2 \pi i z}, q=e^{2 \pi i \tau}$. We often use the following abbreviated notation

$$
\vartheta_{k}(z):=\vartheta_{k}(z, \tau), \quad \vartheta_{k}:=\vartheta_{k}(0, \tau)
$$

The Dedekind eta function is defined as

$$
\eta(\tau):=q^{1 / 24} \prod_{n=1}^{\infty}\left(1-q^{n}\right)
$$

The Eisenstein series are given by

$$
E_{2 n}(\tau)=1+\frac{2}{\zeta(1-2 n)} \sum_{k=1}^{\infty} \frac{k^{2 n-1} q^{k}}{1-q^{k}}
$$

We often abbreviate $\eta(\tau), E_{2 n}(\tau)$ as $\eta, E_{2 n}$ respectively.

The Weierstrass $\wp$-function is defined as

$$
\wp(z)=\wp\left(z ; 2 \omega_{1}, 2 \omega_{3}\right):=\frac{1}{z^{2}}+\sum_{(m, n) \in \mathbb{Z}_{\neq(0,0)}^{2}}\left[\frac{1}{\left(z-\Omega_{m, n}\right)^{2}}-\frac{1}{\Omega_{m, n}{ }^{2}}\right],
$$

where $\Omega_{m, n}=2 m \omega_{1}+2 n \omega_{3}$. We also introduce the following notation

$$
e_{k}:=\wp\left(\omega_{k}\right) \quad(k=1,2,3)
$$

with

$$
\omega_{1}+\omega_{2}+\omega_{3}=0, \quad \frac{\omega_{3}}{\omega_{1}}=\tau
$$


In the main text we use the following identities

$$
\begin{aligned}
\wp^{\prime}(z)^{2} & =4 \wp(z)^{3}-\frac{\pi^{4}}{12 \omega_{1}^{4}} E_{4 \wp}(z)-\frac{\pi^{6}}{216 \omega_{1}^{6}} E_{6} \\
& =4\left(\wp(z)-e_{1}\right)\left(\wp(z)-e_{2}\right)\left(\wp(z)-e_{3}\right) \\
& =\frac{\pi^{6}}{\omega_{1}^{6}} \eta^{12} \prod_{k=1}^{3} \frac{\vartheta_{k+1}\left(\frac{z}{2 \pi}\right)^{2}}{\vartheta_{1}\left(\frac{z}{2 \pi}\right)^{2}} .
\end{aligned}
$$

Open Access. This article is distributed under the terms of the Creative Commons Attribution License (CC-BY 4.0), which permits any use, distribution and reproduction in any medium, provided the original author(s) and source are credited.

\section{References}

[1] O.J. Ganor and A. Hanany, Small $E_{8}$ instantons and tensionless noncritical strings, Nucl. Phys. B 474 (1996) 122 [hep-th/9602120] [InSPIRE].

[2] N. Seiberg and E. Witten, Comments on string dynamics in six-dimensions, Nucl. Phys. B 471 (1996) 121 [hep-th/9603003] [INSPIRE].

[3] A. Klemm, P. Mayr and C. Vafa, BPS states of exceptional noncritical strings, hep-th/9607139 [INSPIRE].

[4] J. Minahan, D. Nemeschansky, C. Vafa and N. Warner, E-strings and $N=4$ topological Yang-Mills theories, Nucl. Phys. B 527 (1998) 581 [hep-th/9802168] [INSPIRE].

[5] O.J. Ganor, D.R. Morrison and N. Seiberg, Branes, Calabi-Yau spaces and toroidal compactification of the $N=1$ six-dimensional $E_{8}$ theory, Nucl. Phys. B 487 (1997) 93 [hep-th/9610251] [INSPIRE].

[6] T. Eguchi and K. Sakai, Seiberg-Witten curve for the E-string theory, JHEP 05 (2002) 058 [hep-th/0203025] [INSPIRE].

[7] T. Eguchi and K. Sakai, Seiberg-Witten curve for E-string theory revisited, Adv. Theor. Math. Phys. 7 (2004) 419 [hep-th/0211213] [INSPIRE].

[8] K. Sakai, Topological string amplitudes for the local half K3 surface, arXiv:1111.3967 [INSPIRE].

[9] M. Alim and E. Scheidegger, Topological strings on elliptic fibrations, arXiv:1205.1784 [INSPIRE].

[10] A. Klemm, J. Manschot and T. Wotschke, Quantum geometry of elliptic Calabi-Yau manifolds, arXiv:1205.1795 [INSPIRE].

[11] B. Haghighat, A. Iqbal, C. Kozcaz, G. Lockhart and C. Vafa, M-strings, arXiv:1305.6322 [INSPIRE].

[12] M.-X. Huang, A. Klemm and M. Poretschkin, Refined stable pair invariants for $E-, M$ - and [p,q]-strings, JHEP 11 (2013) 112 [arXiv:1308.0619] [INSPIRE].

[13] K. Sakai, Seiberg-Witten prepotential for E-string theory and random partitions, JHEP 06 (2012) 027 [arXiv: 1203.2921] [INSPIRE]. 
[14] K. Sakai, Seiberg-Witten prepotential for E-string theory and global symmetries, JHEP 09 (2012) 077 [arXiv: 1207.5739] [INSPIRE].

[15] T.J. Hollowood, A. Iqbal and C. Vafa, Matrix models, geometric engineering and elliptic genera, JHEP 03 (2008) 069 [hep-th/0310272] [INSPIRE].

[16] N.A. Nekrasov, Seiberg-Witten prepotential from instanton counting, Adv. Theor. Math. Phys. 7 (2004) 831 [hep-th/0206161] [InSPIRE].

[17] N. Nekrasov and A. Okounkov, Seiberg-Witten theory and random partitions, hep-th/0306238 [INSPIRE].

[18] H. Nakajima and K. Yoshioka, Instanton counting on blowup. 1., Invent. Math. 162 (2005) 313 [math/0306198] [INSPIRE].

[19] N. Seiberg and E. Witten, Electric-magnetic duality, monopole condensation and confinement in $N=2$ supersymmetric Yang-Mills theory, Nucl. Phys. B 426 (1994) 19 [Erratum ibid. B 430 (1994) 485-486] [hep-th/9407087] [INSPIRE].

[20] N. Seiberg and E. Witten, Monopoles, duality and chiral symmetry breaking in $N=2$ supersymmetric QCD, Nucl. Phys. B 431 (1994) 484 [hep-th/9408099] [INSPIRE].

[21] J. Minahan, D. Nemeschansky and N. Warner, Partition functions for BPS states of the noncritical $E_{8}$ string, Adv. Theor. Math. Phys. 1 (1998) 167 [hep-th/9707149] [INSPIRE].

[22] F. Benini, S. Benvenuti and Y. Tachikawa, Webs of five-branes and $N=2$ superconformal field theories, JHEP 09 (2009) 052 [arXiv:0906.0359] [INSPIRE].

[23] L. Bao, V. Mitev, E. Pomoni, M. Taki and F. Yagi, Non-lagrangian theories from brane junctions, arXiv:1310.3841 [INSPIRE].

[24] H. Hayashi, H.-C. Kim and T. Nishinaka, Topological strings and $5 d T_{N}$ partition functions, arXiv:1310.3854 [INSPIRE]. 